shops, healthcare, and usual activities due to restrictions. Findings emphasize the issue of loneliness and isolation for some of the participants, especially those living alone, as well as fear of the virus, with restrictions leading to a loss of purpose for some, along with related effects on mental health (e.g. worry, anxiety). For some, these issues appeared to link to a reprioritisation of their behaviours (for example, exercise and diet were deprioritised). Coping strategies used by participants included finding ways to keep busy and to do physical activity safely, maintaining social contact online or by telephone, and having an optimistic or positive outlook, a 'do what you can' attitude.

Conclusion Analysis is ongoing and will further aim to explore how older people's experiences and behaviours might have changed over the duration of the pandemic. The findings from this study could improve understanding of how community-living older adults could be supported to be more resilient in the face of a variety of changing circumstances that might impact their health and well-being.

\section{P53 EFFECTS OF COVID-19 PUBLIC HEALTH SAFETY MEASURES ON BIRTHS IN SCOTLAND BETWEEN MARCH AND MAY 2020}

${ }^{1}$ Louise Marryat*, ${ }^{2}$ Lydia Speyer, ${ }^{2}$ Bonnie Auyeung. ${ }^{1}$ School of Health Sciences, University of Dundee, Dundee, UK; ${ }^{2}$ School of Psychology, University of Edinburgh, Edinburgh, UK

\subsection{6/jech-2021-SSMabstracts. 141}

Background To combat the wide-spread transmission of COVID-19, many countries, including the Scotland, have imposed nationwide lockdowns. This included changes to NHS maternity and ante/postnatal services and policies. Little is known about how these lockdown measures affect pregnant mothers and their offspring. This study aimed to explore the impact of COVID19 public health safety measures on birth outcomes in Scotland.

Methods Using routinely collected health data on pregnancy and birth in Scotland, this study compares all births $(\mathrm{N}=$ 11220) between March and May 2020 to births in the same period in $2018(\mathrm{~N}=12428)$ to investigate the potential effects of lockdown measures introduced in Scotland in spring 2020. A variety of maternal and infant outcomes were analysed: induction of labour (yes, no), mode of delivery (unassisted vaginal delivery, planned caesarean section, emergency caesarean section, other (e.g. use of forceps)), analgesia during labour (none, gas and air, opioids, epidural, spinal anaesthetic, general anaesthetics, other), birth outcome (livebirth, stillbirth, infant death), APGAR score $(<3,4-<7,>7)$, age of gestation, birthweight, length of hospital stay, and feeding method on discharge (breastfeeding, formula, mixed feeding, other). Data were analysed using Mann-Whitney U tests for continuous outcomes and chi-square tests of independence for nominal outcomes. If the chi-square test was significant $(\alpha<0.05)$, post-hoc tests (Fisher's exact tests) were conducted to examine all possible comparisons. These were corrected for multiple comparisons using Bonferroni adjustment.

Results Overall there were few statistically significant differences between births in 2018 and 2020: women in 2020 were more likely to require spinal anaesthetics compared to using no pain relief air $(P=.035)$, gas and air $(P<.001)$ or opioids $(P<.001)$ as well as more likely to have an epidural compared to using gas and air $(P<.001)$ or opioids $(P<$ .001). Mothers giving birth during the pandemic also tended to combine breastfeeding and formula-feeding on discharge $\left(\chi^{2}(3)=19.09, p<.001\right)$ : post-hoc tests revealed that women were more likely to combine breastfeeding with formula-feeding than to exclusively breastfeed $(P<.001)$ or exclusively formula-feed $(P<.001)$. Finally, women stayed in hospital for fewer days $(Z=-10.90, p<.001)$ and more often had an epidural or a spinal anaesthetic compared to women giving birth in 2018.

Conclusion In conclusion, findings of the current study suggest that lockdown measures implemented in Scotland as a response to the COVID-19 pandemic had limited effects on maternal and neonatal outcomes. While these findings are reassuring, future research is needed to gain better insights into the impact of COVID-19 and associated lockdown measures on maternal and child health.

\section{P54 PHASE 2 OF THE NORWICH COVID-19 TESTING INITIATIVE: AN EVALUATION}

${ }^{1}$ Tara Berger Gillam*, ${ }^{2}$ Jeannette Chin, ${ }^{3}$ Karim Gharbi, ${ }^{5}$ Mark Hitchcock, ${ }^{4}$ Rose Davidson, ${ }^{3}$ Neil Shearer, ${ }^{1}$ Nick Steel. ${ }^{1}$ Health Services and Primary Care Research Group, University of East Anglia, Norwich, UK; ${ }^{2}$ School of Computing Sciences, University of East Anglia, Norwich, UK; ${ }^{3}$ Earlham Institute, Norwich, UK; ${ }^{4}$ Faculty of Medicine and Health Sciences, University of East Anglia, Norwich, UK; ${ }^{5}$ UEA Health and Social Care Partners, University of East Anglia, Norwich, UK

\subsection{6/jech-2021-SSMabstracts. 142}

Background Phase 2 of the Norwich Testing Initiative (NTI2) was a SARS-CoV-2 PCR testing programme at the University of East Anglia, which ran from September to December 2020. It aimed to identify asymptomatic COVID-19 infections and limit outbreaks on campus. The NTI2 evaluation explored testing uptake, positivity rates, isolation compliance and the links between viral load and symptoms.

Methods All staff and students $(21,762)$ were eligible for testing. Users registered for the programme using a web application, which collected personal, demographic and location data. Users collected tests from a central location on campus, selfadministered a nasopharyngeal swab and returned tests to a drop-off point. PCR testing was conducted by two laboratories. All those with a positive result were contacted by telephone for self-isolation advice and support. At this point symptom, isolation and location data were collected. The programme used a broader definition of COVID-19 symptoms than that used by NHS Test and Trace. Data were encrypted and stored in a data warehouse. Microsoft Excel was used to collate, clean and analyse the data.

Results 188 of 6537 (2.9\%) users tested positive for COVID19. The majority (82\%) were students aged 18-24 years. Positivity rates were highest in students $(3.5 \%)$, those living on campus (6.5\%) and BAME groups (4\%). There was clustering of cases in university halls: positivity rates in halls varied between $0 \%$ and $31 \%$, with $18 \%$ of halls containing over half the cases on campus. Positivity rates spiked near the beginning of the programme and then declined over the course of the term. 99/187 (53\%) cases reported symptoms of COVID-19. $37 / 99(35 \%)$ symptomatic cases were not isolating. There was no significant relationship between PCR $C_{t}$ values and selfreported symptoms.

Conclusion NTI2 may have contributed to a decline in cases of COVID-19 during the testing period, as the incidence of 
COVID-19 in the local area increased during this period. Transmission within halls of residence may have contributed to high positivity rates on campus. A high proportion of users reported symptoms of COVID-19 infection, despite this being an asymptomatic testing programme. Over a third of symptomatic cases were not isolating prior to receiving their result . These findings suggest that testing and isolating messaging may not be reaching the student population. Those with symptoms had a similar viral load to those without and may be similarly infectious.

\section{P55 LOW INCOME PARENTS' PERSPECTIVES AND EXPERIENCES OF ENGAGING WITH EARLY YEARS HEALTH PROFESSIONALS ABOUT FINANCIAL CHALLENGES AND INCOME MAXIMISATION}

${ }^{1}$ Flora Douglas*, ${ }^{1}$ Emma Maclver, ${ }^{2}$ Tracy Davis. ${ }^{1}$ School of Nursing, Midwifery and Paramedic Practice, Robert Gordon University, Aberdeen, UK; ${ }^{2}$ Public Health Directorate, NHS Grampian, Aberdeen, UK

\subsection{6/jech-2021-SSMabstracts. 143}

Background Since the introduction of the Child Poverty Act (2017) in Scotland, all health visitors, midwives and family nurses in Scotland are expected to screen and offer a financial advice referral to at-risk pregnant women and parents/carers of families with children under five in Scotland. The so-called 'Financial Inclusion Pathway' (FIP) emerged in 2019 as one of a number of strategies intended to tackle child poverty. At this early implementation stage, little is known about parents' perspectives about the acceptability or impact of this initiative in relation to its aim.

Methods In 2020, low income parents with young children living in Aberdeen City were invited to take part in a qualitative study that aimed to determine any challenges they might face engaging with the FIP policy in practice, and their perspectives about financial discussions with professionals that could help to increase household incomes through this approach.One-to-one semi-structured telephone interviews lasting between 30-40 minutes took place during July and August 2020. Interviews were fully transcribed and thematically analysed.

Results Ten women, ranging from ages 20-41 years. Each participant had between one and five child(ren), ranging in age from 2 to 18 years and all had one child under school-age. All lived in multiply deprived postcode areas within Aberdeen City. Three main themes emerged: i. difficulties associated with discussing financial concerns; ii. how to talk about the issue; iii. intervention utility. Within each of these three main themes, nine-sub themes were apparent, ranging from those indicating potential unintended negative consequences, as well as intervention benefits.

Discussion Most participants considered health visitors to be a potentially good source of help about financial challenges; they were less clear about midwives' role here. However, parents' perceptions of the problems they may face associated with disclosing financial difficulties to health professionals, is a distinct barrier to conversations that could lead to a financial advice referral. Given the levels of unclaimed benefit in the UK, this is important to note. Establishing trust and rapport, careful and sensitive enquiry, and positive framing of financial maximisation were recommended to aid discussion of financial challenges. These findings are important given the current and predicted economic impact of the COVID-19. The study is limited by its sample size and recruitment site, but provides valuable insights to inform a larger scale investigation. The study also indicates the importance of understanding health professionals' associated views and experiences.

\section{P56 CHILDREN'S HEALTH IN CARE IN SCOTLAND (CHICS) STUDY - POPULATION-WIDE LINKED ADMINISTRATIVE DATA ANALYSIS COMPARING HEALTH OUTCOMES FOR CARE EXPERIENCED AND CHILDREN IN THE GENERAL POPULATION, 2009-2016}

${ }^{1}$ Mirjam Allik*, 'Denise Brown, ${ }^{1}$ Alastair Leyland, ${ }^{2}$ Marion Henderson. ${ }^{1}$ SPHSU, University of Glasgow, Glasgow, UK; ${ }^{2}$ Social Work and Social Policy, University of Strathclyde, Glasgow, UK

\subsection{6/jech-2021-SSMabstracts. 144}

Background There is little quantitative evidence on how the health of care experienced children compares to children in the general population in Scotland or the UK. Invariably, the evidence that is available suggests that care experience is related to poorer health, but often this is based on small sample sizes or without comparison to children who have not been in care. The CHiCS study was set up to provide the first population-wide evidence on health outcomes (mortality, hospitalisation, prescribing and pregnancy rates) of care experienced children (CEC) compared to children in the general population (CGP).

Methods We undertook an individual level linkage of the Children Looked After in Scotland (CLAS) return to the Pupil Census (PC), Scottish Morbidity Records (SMR00, 01, 02, 04), Accidents and Emergency data, Prescribing Information System, and Death and Birth Registrations from August 2009 to July 2016. The CEC cohort was defined as all children who were on the 2009/2010 CLAS return and the CGP cohort was defined as children who were on the 2009 PC but had not been in care. Age-standardized rates by area deprivation will be calculated for various outcomes for both cohorts to provide the first national evidence on the health of looked after children. Event history analysis will be used on matched cohorts to investigate the impact of placement histories and parental socioeconomic status (SES) on health.

Results The data became available for analysis in December 2020. The initial results show that CEC have, on average, lower SES and live in areas of higher deprivation, they also experienced higher average rates of mortality, prescriptions, and all types of hospitalisations during the study period (not adjusted). There are also substantial differences in the types of prescriptions and reasons for hospital admission between the cohorts, e.g. CEC are more likely to be prescribed antidepressant and ADHD medication and have almost three times as many psychiatric outpatient appointments (not adjusted). Additional results will be submitted before the June 28th deadline. We expect the results adjusted for confounders to show substantial differences in the frequency and reasons for contact with health services between the two cohorts.

Results Adverse childhood experiences have a profound negative impact on health and these effects last long into adulthood. Identifying how care experiences are related to health will provide evidence to develop interventions for health improvement and to reduce inequalities. Interventions in childhood are among the most effective in improving long-term health and are cost-effective. 